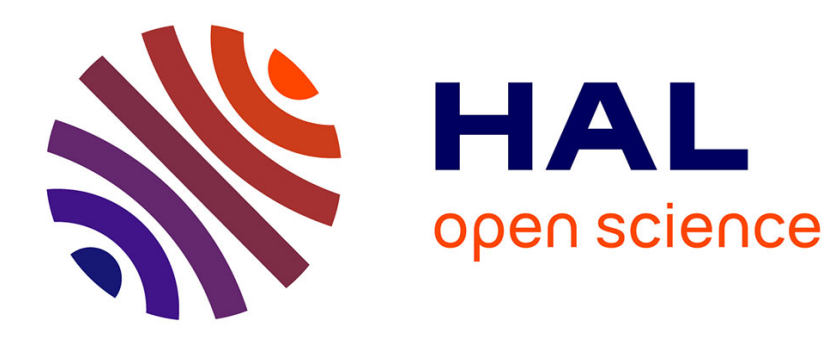

\title{
Le support des réseaux mobiles dans IPv6
}

Thierry Ernst

\section{To cite this version:}

Thierry Ernst. Le support des réseaux mobiles dans IPv6. Revue des Sciences et Technologies de l'Information - Série TSI: Technique et Science Informatiques, 2006, 25 (5), pp.573-598. inria00102315

\section{HAL Id: inria-00102315 https://hal.inria.fr/inria-00102315}

Submitted on 29 Sep 2006

HAL is a multi-disciplinary open access archive for the deposit and dissemination of scientific research documents, whether they are published or not. The documents may come from teaching and research institutions in France or abroad, or from public or private research centers.
L'archive ouverte pluridisciplinaire HAL, est destinée au dépôt et à la diffusion de documents scientifiques de niveau recherche, publiés ou non, émanant des établissements d'enseignement et de recherche français ou étrangers, des laboratoires publics ou privés. 


\title{
Le support des réseaux mobiles dans IPv6
}

\author{
Thierry Ernst \\ INRIA Rocquencourt, Domaine de Voluceau B.P. 105 \\ 78153 Le Chesnay Cedex FRANCE \\ thierry.ernst@inria.fr
}

RÉSUMÉ. Les réseaux d'accès ou les réseaux de senseurs déployés entre autres dans les transports ont pour particularité d'être connectés à l'Internet via un ou plusieurs routeurs qui changent fréquemment de point d'ancrage dans la topologie Internet, ce qui entraîne la rupture des sessions ouvertes. Sans un mécanisme de gestion de la mobilité des réseaux, il n'est pas permis d'envisager le développement attendu des applications de contrôle ou multimédia dans les réseaux embarqués car ce type d'application exige une connexion permanente et ininterrompue à Internet. Les travaux traditionnels dans le domaine de la gestion de la mobilité, notamment Mobile IPv6, permettent aux stations mobiles prises individuellement de maintenir leurs sessions ouvertes. En revanche, ils ne permettent pas de les maintenir pour les stations fixes situées derrière un routeur mobile ni pour le réseau mobile pris dans son ensemble. Cet article se propose donc de faire le tour des travaux portant sur la mobilité des réseaux dans IPv6. Nous présentons les usages, les besoins, la problématique et nous faisons le point sur les travaux conduits par le groupe NEMO qui a été créé au sein de l'IETF. Nous terminons cette étude par les éléments prospectifs portant sur l'optimisation du routage.

ABSTRACT. Traditional work turning around mobility support has usually focused on host mobility, i.e. single terminals which change their point of attachment in the Internet topology, whereas the most current type of mobility in IPv6 is probably going to be network mobility, i.e. entire IPv6 networks which border routers change their point of attachment to the Internet topology. Mobile networks are expected to be found in access networks deployed in public transportation, and networks of sensors installed in vehicles. Without specific support mechanisms, changing the point of attachment results into broken sessions. Protocols such as Mobile IPv6 designed to support host mobility are either inappropriate or inefficient to support network mobility. Extensions to Mobile IPv6 have therefore been proposed and are currently discussed in the IETF NEMO working group. The purpose of this paper is to explain the problem caused by network mobility in IPv6 and to overview the current activities of this promising topic.

MOTS-CLÉS : support de la mobilité des réseaux, réseaux mobiles, NEMO, IPv6, Mobile IPv6.

KEYWORDS: network mobility, mobile network, NEMO, IPv6, Mobile IPv6.

RSTI - TSI. Volume 25 - nº 5/2006, pages 573 à 597 


\section{Introduction}

Par mobilité dans Internet, on considère généralement le déplacement dans la topologie Internet d'un équipement de type ordinateur ou téléphone portable. Or, la notion de mobilité peut s'étendre aux réseaux eux-mêmes dès lors que les réseaux dans lesquels sont déployés ces équipements deviennent eux-mêmes mobiles. C'est le cas pour les réseaux de capteurs embarqués dans les véhicules, les réseaux d'accès déployés dans les transports, ou les réseaux personnels (PANs). Le besoin de connecter ces réseaux à Internet est suscité soit par les fabricants de véhicules, soit par les compagnies de transport, soit par les usagers eux-mêmes. Ceci permet alors la collecte de données et le déploiement d'applications multimédia en tout lieu et à tout instant.

Tout équipement, qu'il soit fixe ou mobile par rapport au réseau dans lequel il s'attache, est donc en mesure de se déplacer géographiquement et topologiquement. Le déplacement géographique peut impliquer le changement de technologie d'accès, donc de qualité de service, de bande passante, de règles d'usages (contrôle d'accès), et une vulnérabilité accrue face aux failles de sécurité. Le déplacement géographique a en général pour conséquence un déplacement dans la topologie Internet ce qui implique un changement d'adresse IP. Or, l'adresse IP est utilisée à la fois pour déterminer la position de l'équipement dans la topologie Internet, et pour identifier les sessions établies avec cet équipement. Un mécanisme de gestion de la mobilité est alors indispensable pour fournir une connectivité Internet permanente sans rompre les communications en cours suite à ce changement d'adresse IP.

Les travaux dans le domaine de la gestion de la mobilité dans IPv6 se sont dans un premier temps exclusivement consacrés au support des stations mobiles, c'est-àdire les terminaux changeant individuellement de point d'ancrage dans la topologie Internet. En revanche, les travaux se préoccupant des problèmes spécifiques liés au déplacement simultané d'un ensemble d'équipement regroupés en réseau n'ont réellement vu le jour que récemment.

Le but de cet article est donc de mettre l'accent sur la notion de réseau mobile qui, étant relativement récente, n'est pas forcément connue de tous. Dans un premier temps, nous décrivons quelques usages possibles des réseaux mobiles (section 2) puis nous présentons quelques généralités sur l'organisation de l'Internet, l'adressage et la problématique de la mobilité liée au modèle d'adressage dans TCP/IP (section 3). Partant de là, nous discutons des caractéristiques et des besoins des réseaux mobiles (section 4) avant de dresser l'état d'avancement des travaux de l'IETF (Internet Engineering Task Force) permettant le support de la mobilité des réseaux, notamment au sein du groupe de travail NEMO (section 5). S'en suit une revue des travaux connus à ce jour pour résoudre la question de l'optimisation du routage, classés selon le type d'approche (section 6). Avant de conclure cet article, nous présenterons quelques projets et implémentations des mécanismes du support des réseaux mobiles (section 7).

Cette étude est exclusivement consacrée à IPv6 (Deering et al., 1998; Cizault, 2005) car il n'est pas envisageable de déployer de tels réseaux mobiles dans IPv4, d'autant plus qu'aucun travail concret n'y est répertorié. Les termes utilisés dans cet 
article sont essentiellement les traductions des termes extraits de (Manner et al., 2004) qui offre une terminologie appliquée à la mobilité relativement complète, et de (Ernst et al., 2006) qui définit une terminologie spécifique à la problématique de la mobilité des réseaux. Le lecteur intéressé par les aspects techniques se référera à la page web non officielle du groupe $\mathrm{NEMO}^{1}$ pour retrouver l'ensemble des documents relatifs aux travaux de l'IETF. Quant à ceux plutôt intéressés par les aspects prospectifs, ils porteront un regard attentif sur les sections 5.5 et 6 .

\section{Les usages}

De nombreux usages des réseaux mobiles sont envisagés. Ceux-ci incluent en particulier les réseaux personnels (Personal Area Networks, ou PANs) et les réseaux déployés dans les véhicules (Vehicular Area Network, ou VANs), c'est-à-dire les réseaux de capteurs et les réseaux d'accès :

- Cas des réseaux de capteurs : ceux-ci sont déployés dans les véhicules (avions, trains, bateaux, voitures). Certains ont besoin d'interagir avec des serveurs dans l'Internet, par exemple pour assurer la transmission de données nécessaires à la navigation, pour procéder à la maintenance et au contrôle de l'état du véhicule, etc. Un autre exemple, encore futuriste, est celui des vêtements intelligents dans lesquels sont incorporés des capteurs (humidité, température, rythme cardiaque, tension artérielle, etc.) permettant entre autres le contrôle en temps réel de l'état de santé d'un patient.

- Cas des réseaux d'accès : ceux-ci sont déployés dans les transports publics (bus, trains et taxis) et permettent d'offrir une borne d'accès Internet aux passagers. L'exemple le plus typique est celui d'un véhicule disposant d'un accès Internet par le biais de technologies sans fil variées (cellulaire, IEEE 802.11, Bluetooth, satellite) afin d'améliorer la sécurité et la navigation et de fournir du contenu multimédia aux passagers, tout ceci en temps réel (Kellerer et al., 2001; Ernst et al., 2002; Lach et al., 2003). Ce type de réseau embarqué a pour caractéristique principale le changement fréquent de point d'ancrage, non seulement à cause de sa vitesse de déplacement, mais aussi à cause de la variété des technologies offertes en fonction du pays, de la densité de la circulation, de la densité d'urbanisation, etc. Un autre exemple tout aussi démonstratif est celui d'une compagnie de transport ferroviaire ou aérienne offrant un accès Internet permanent et ininterrompu à ses passagers. Cet accès pourra non seulement permettre aux passagers de se connecter sur un site distant, de télécharger de la musique et de la vidéo depuis n'importe quel fournisseur de service, ou de surfer sur la toile sans interruption de service en utilisant les appareils proposés par la compagnie, mais aussi de s'y connecter en utilisant leur propre ordinateur portable ou téléphone. Ce scénario est d'ailleurs mentionné depuis longtemps dans (Tanenbaum, 1996), section 5.15; (Partridge, 1994), sections 1.2.4 et 5.5.8; (Perkins, 1998), section 5.12; (Solomon, 1998), section 11.2, (Perkins, 2002) section 4.5. Deux expériences de ce type ont déjà été conduites en 2002 par deux compagnies ferroviaires distinctes de la

1. Page additionnelle du groupe NEMO : http ://www.mobilenetworks.org/nemo 
banlieue de Tokyo. La compagnie JR permettait, à bon escient pendant la durée de la réunion de l'IETF à Yokohama en juillet 2002, aux passagers du premier wagon de connecter leur ordinateur portable à Internet via une connexion IEEE $802.11 \mathrm{~b}$ sur un boîtier IPv6 embarqué dans le train reliant Yokohama à l'aéroport Narita ${ }^{2}$. Dans l'expérience, un utilisateur disposant d'un accès sans fil 802.11b sur son ordinateur portable pouvait se connecter à Internet via ce boîtier, lui-même connecté à l'Internet via une connexion IEEE 802.11b lors des arrêts en gare, et via le réseau cellulaire pendant les déplacements. La deuxième expérience, conduite avec la compagnie Odakyu en utilisant le code développé au sein de Jun Murai Lab, Keio University, était plus modeste, et avait pour but de connecter un routeur à Internet, et de tester les changements d'interface entre IEEE $802.11 \mathrm{~b}$ (lors des arrêts en terminus), et le réseau cellulaire (pendant les déplacements).

- Cas spécifique de l'automobile : le domaine des systèmes embarqués à bord des véhicules jouit actuellement d'un grand engouement un peu partout dans le monde. Il existe en effet de nombreux projets généralement regroupés sous la dénomination ITS (Intelligent Transportation Systems) qui visent à améliorer la sécurité routière, la navigation, la conduite, la disponibilité et l'entretien des véhicules. Ceci nécessite la transmission de données entre véhicules, ou entre un véhicule et l'infrastructure routière ou l'Internet. On citera en autres le projet RNRT $^{3}$ Everyware labellisé en octobre 2002 comprenant notamment Renault et France Telecom; le consortium InternetITS ${ }^{4}$, un projet de grande envergure réunissant au Japon une centaine d'industriels et d'universitaires travaillant en relation étroite avec les fabriquants d'automobiles (le système de communication mis en place est en fait celui développé par le projet ICAR décrit dans la section 7); et le projet FleeNet.

- Cas spécifique de l'aviation : dans ce cas spécifique, outre son utilisation par les passagers, l'Internet peut aussi servir à la maintenance et à la gestion de la flotte par la compagnie aérienne, ainsi qu'à échanger les données destinées à la navigation entre l'avion et les tours de contrôle aérien. Ce dernier scénario a justement fait l'objet de recherches de la part d'Eurocontrol (une organisation européenne pour la sécurité de la navigation aérienne) (Robert, 1999; Quinot, 1998), ainsi que de la part d'Airbus (AFIS) et Boeing (Corenet). Le cas de l'avion est particulier car celui-ci change rarement de point d'ancrage. Au cours d'un vol international, l'avion est connecté via un satellite géo-stationnaire au-dessus des océans, via un lien radio au-dessus des terres (Terry, 2004), et via un WLAN de type 802.11 lors des stationnements. Un service commercial (connexion) développé par Boeing et permettant l'accès Internet en vol a été lancé sur certaines compagnies en $2004^{5}$.

- Cas des réseaux personnels : les PANs sont des réseaux constitués d'un ensemble d'appareils électroniques de petite taille (cardio-fréquence-mètre, montre, téléphone cellulaire, assistant personnel, appareil photo digital, etc.) portés par les personnes. De nombreux scénarii d'utilisation des PANs peuvent être imaginés, notam-

2. JR Narita Express (NEX) : http ://itpro.nikkeibp.co.jp/free/ITPro/OPINION/20020702/1/

3. RNRT : Réseau National de Recherche en Télécommunications, France.

4. InternetITS : http ://www.internetITS.org

5. voir la présentation de Boeing lors de l' assemblée plénière du $62^{e}$ IETF en mars 2005. 
ment pour des applications liées à la sécurité civile (police, pompiers) (Boot, 2002), à la médecine (Ernst, 2004) et bien entendu aussi à l'armée. Par exemple, un fauteuil roulant, un sac, ou un vélo équipé d'un PAN pourrait permettre, à un handicapé moteur, à une personne ayant des déficiences mentales, ou à un sportif, d'être suivi à distance et en temps réel (par un médecin, la famille, la cellule antidopage, etc.) et d'appeler automatiquement les secours en cas de défaillance, voire de prodiguer des conseils immédiats à la victime. Ces scénarii sont étudiés à but démonstratif dans le projet Nautilus6 (voir section 7) et pourraient aussi bien répondre aux besoins de la police, des pompiers, des journalistes, etc.

\section{Généralités sur la mobilité des stations et des réseaux}

Dans cette section, nous expliquons brièvement la problématique de la mobilité liée au modèle d'adressage de TCP/IP :

- Organisation de l'Internet et modèle adressage : l'Internet est une agglomération de réseaux partitionnés en plusieurs domaines. Au niveau logique, un domaine représente d'ordinaire une université, une entreprise, ou un fournisseur de service. Un domaine peut lui-même être divisé en sites, par exemple chaque campus au sein d'une université ou chaque usine au sein d'une entreprise. Au niveau physique, chaque site ou domaine se décompose en sous-réseaux, eux-mêmes constitués d'un lien (e.g. Ethernet) et de l'ensemble des nœuds se trouvant sur ce même lien. Les nœuds sont de deux types. Ceux qui relient un sous-réseau à un autre sont des routeurs, les autres de simples stations. A chaque sous-réseau, correspond un préfixe IP qui permet d'identifier la position du sous-réseau dans la hiérarchie de l'Internet. Tous les nœuds ayant une interface sur un sous-réseau donné ont donc une adresse IP correspondant au préfixe de ce sous-réseau. Cette adresse identifie à la fois la position topologique du nœud, et le nœud lui-même ${ }^{6}$. L'adresse IP est donc intrinsèquement liée à la position du nœud dans la topologie Internet. En résumé, un réseau est un ensemble de sousréseaux, c'est-à-dire de liens et de nœuds partageant le même préfixe IP et connectés à l'Internet par le biais d'un ou plusieurs routeurs externes.

- Problématique de la mobilité : un nœud mobile (MN) est un nœud qui change son point d'ancrage dans la topologie Internet, c'est-à-dire qui se déplace d'un sousréseau à un autre. Dans le cas des stations, nous parlons de station mobile, dans le cas de routeur, de routeur mobile (MR). Les routeurs d'accès (ARs) sont les routeurs qui desservent les liens où les noeuds mobiles peuvent prendre ancrage. Le point d'ancrage initial est appelé sous-réseau mère tandis que chaque point d'ancrage subséquent est appelé sous-réseau visité. Le problème posé par la mobilité vient essentiellement du modèle d'adressage de TCP/IP qui confond le rôle d'identifiant d'interface de l'adresse IP, et son rôle d'identifiant de la localisation dans la topologie Internet qui est hiérarchisée. Si un nœud change d'emplacement dans la topologie Internet

6. En fait, l'adresse IP identifie l'interface d'un nœud, mais pour simplifier nous nous contentons souvent de dire que l'adresse identifie le nœud. 


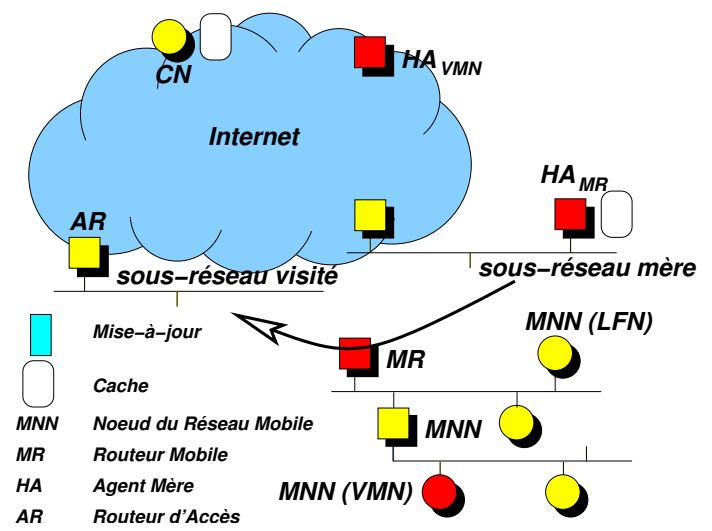

Figure 1. Terminologie pour les Réseaux Mobiles

l'adresse IP qui identifie l'interface qui change d'emplacement doit changer. Ce changement d'adresse a pour conséquence de rompre les sessions ouvertes qui se servent de l'adresse IP comme identificateur tandis que le changement d'emplacement nécessite un re-routage. Le support de la mobilité a donc pour but, d'une part de définir un mécanisme permettant de maintenir les sessions ouvertes lors des déplacements, et d'autre part de déterminer la nouvelle position du noud dans la topologie (localisation et routage). Ceci se fait généralement au prix de messages de contrôle (signalisation). Pour de plus amples détails, nous invitons le lecteur à se référer par exemple à (Ernst, 2001) (chapitre 2) ou (Soliman, 2004) (chapitre 1).

- Réseau mobile : un réseau mobile est défini comme un sous-réseau ou un ensemble de sous-réseaux connectés à l'Internet par l'intermédiaire d'un ou plusieurs routeurs mobiles qui changent leurs points d'ancrage (AR) à l'Internet. Les termes MNNs (Mobile Network Node) et CN (Correspondent Node) désignent respectivement tout nœud localisé à l'intérieur du réseau mobile derrière le MR, et tout nœud communiquant avec un ou plusieurs MNNs. Les interfaces d'un MR connectées sur un sous-réseau mère ou un sous-réseau visité sont nommées interfaces externes tandis que toutes les autres interfaces sont nommées interfaces internes. Toute interface devant disposer d'une adresse sur le lien auquel elle est raccrochée, le préfixe de l'interface externe sera le même que celui du sous-réseau mère ou celui du sous-réseau visité, tandis que le préfixe publié dans le réseau mobile (MNP ou Mobile Network Prefix) servira à configurer les adresses de l'interface interne du MR et de tous les MNNs. Ces termes sont illustrés sur la figure 1 qui montre un réseau mobile se déplaçant de son sous-réseau mère vers un autre sous-réseau. 


\section{Caractéristiques et besoins au niveau de l'architecture IPv6}

Les scénarii présentés dans la section 2 justifient tous le besoin d'un certain nombre d'équipements interconnectés entre eux, et le besoin d'un accès Internet direct pour certains, donc un réseau embarqué de type IP, c'est-à-dire un réseau mobile. Ils démontrent aussi que les réseaux mobiles peuvent avoir des caractéristiques et donc des besoins très différents d'un cas à l'autre :

- Taille : les réseaux mobiles peuvent être de taille variable, allant de l'ordre de quelques MNNs dans le cas d'un PAN jusqu'à plusieurs centaines de stations interconnectées par plusieurs routeurs et sous-réseaux dans le cas d'un train. Le nombre de correspondants (CNs), quant à lui, est indépendant du nombre de MNNs, mais peut potentiellement être très grand. Par exemple, une source vidéo émise depuis un véhicule peut avoir à elle seule un nombre de récepteurs du même ordre de grandeur que celui de la multitude de correspondants de l'ensemble des passagers d'un train, même si le second cas semble plus réaliste. Dans un cas comme dans l'autre, un grand nombre de sessions peuvent avoir lieu simultanément, toutes transitant via le MR. Ainsi, la quantité de trafic induit par ou vers un réseau mobile est d'autant plus significative que le nombre de CNs est grand. Pour permettre un passage à l'échelle, il conviendra donc de prendre en considération non seulement le nombre de réseaux mobiles, mais aussi le nombre de correspondants. Ceci nous permet de faire une comparaison avec la gestion de la mobilité pour les stations mobiles où le seul paramètre pris en compte jusqu'à présent est le nombre de stations mobiles.

- Hétérogénéité des MNNs : les nœuds embarqués (MNNs) peuvent être de trois types. Un LFN (Local Fixe Node) est un nœud résidant de manière permanente dans le réseau mobile et ne changeant pas son point d'ancrage (par exemple un capteur de pression des pneus ou de température). Un LMN (Local Mobile Node) est un nœud mobile appartenant au réseau mobile et capable de changer son point d' ancrage dans le réseau mobile, voire de le quitter (e.g. la clef du véhicule), tandis qu'un VMN (Visiting Mobile Node) est un nœud mobile n'appartenant pas au réseau mobile mais capable de s'y attacher (e.g. équipements appartenant aux passagers tels un ordinateur portable ou un PDA).

- Mobilité enchaînée (Nested Mobility) : un réseau mobile pouvant accueillir soit une station mobile, soit un routeur mobile servant lui-même de passerelle à un autre réseau mobile, la mobilité des réseaux peut être récursive. Dans le cas d'un bus servi par un MR et offrant un accès Internet aux stations mobiles (VMN) des passagers, nous avons deux niveaux de mobilité. Dans le cas d'un passager disposant d'un PAN qui à son tour permet l'ancrage d'un VMN, nous devons faire face à trois niveaux de mobilité. Le réseau et les MRs qui connectent l'ensemble à Internet sont respectivement appelés root-NEMO et root-MR. Les autres réseaux (respectivement MRs) se servant d'un root-NEMO pour se connecter à Internet sont nommés sub-NEMO et sub-MRs. Deux niveaux de mobilité sont illustrés sur la figure 1. Le réseau mobile y accueille une station mobile (VMN) issue d'un autre sous-réseau (identifié par $H A_{V M N}$ ).

- Hétérogénéité des réseaux d'accès : au vu de l'exemple de l'automobile qui peut être amenée à se déplacer sur de longues distances, passer d'un milieu urbain 
aux technologies d'accès variées à un milieu rural pauvre en ressources disponibles, changer de pays, etc., nous constatons que d'une part un réseau mobile peut prendre ancrage à l'Internet via des points très éloignés dans la topologie, et d'autre part le faire par le biais de technologies hétérogènes. Il est ainsi raisonnable de considérer le cas où les réseaux mobiles non seulement changent de réseau d'accès, mais certainement aussi de fournisseurs de service ou de domaine administratif (mobilité globale). Dans un tel cas, la sécurisation des données de contrôle, le contrôle d'accès aux ressources du réseau visité, et l'adaptation des applications à la bande passante disponible sont des besoins cruciaux.

- Multidomiciliation (Multihoming) : un réseau mobile est dit multidomicilié lorsqu'il a plusieurs points d'ancrage à Internet, c'est-à-dire lorsqu'il est simultanément connecté à Internet via plusieurs MRs ou lorsqu'un MR a plusieurs interfaces externes, ou plusieurs adresses sur son interface externe. Les motivations et les bénéfices attendus sont les mêmes pour un réseau fixe ou mobile, mais la mobilité rend cette configuration plus fréquente. En effet, une telle configuration permet de pallier aux pannes, de partager les flux, de mettre en place des préférences ou plus simplement de garantir un meilleur accès à l'Internet en faisant appel à plusieurs technologies. Cette possibilité de se connecter par l'intermédiaire d'un ou plusieurs routeurs mobiles disposant au total de plusieurs interfaces externes nécessite de considérer les aspects de changement d'interface et de changement de routeur mobile.

- Interaction entre réseau Ad Hoc et réseau mobile : les réseaux ad hoc sont des réseaux sans infrastructure, dont l'ensemble des nœuds sont des routeurs mobiles, avec ou sans sous-réseau attaché à leur interface interne, et dont la topologie est très dynamique. Les routes de la source à la destination (en règle générale toutes deux situées à l'intérieur du réseau ad hoc) sont calculées dynamiquement. L'hétérogénéité des MNNs peut mener à confondre réseaux mobiles et réseaux ad hoc car il est possible qu'un réseau ad hoc constitue un réseau mobile et inversement qu'un ensemble de réseaux mobile constitue un réseau ad hoc. Par exemple, les passagers d'un train peuvent former un réseau ad hoc. Si la connectivité à Internet est offerte par le biais d'une passerelle à bord du train changeant son point d'ancrage (i.e. un MR), il s'agit d'un réseau mobile constitué de nœuds ad hocs. En revanche, lorsque des réseaux sont embarqués dans des véhicules, et qu'une flotte de véhicules de ce type forme un réseau ad hoc, nous avons un réseau ad hoc constitué de réseaux mobiles (Kellerer $e t$ al., 2001).

- Fréquence distincte de changement du point d'ancrage : à chaque configuration, et selon l'usage, correspond une certaine fréquence de mobilité. Un métro, par exemple, suit une trajectoire déterminée à vitesse déterminée, probablement au sein d'un seul fournisseur d'accès. Les handovers sont donc prévisibles. Un piéton ou une automobile en milieu urbain, en revanche, change de trajectoire, de vitesse, et de réseau d'accès; les handovers sont donc très difficilement prévisibles. Ils seront en revanche semi-prévisibles dans le cas d'une voiture sur autoroute. 


\begin{tabular}{|c|c|c|c|}
\hline Type & Mobilité & Equipements & Besoins Particuliers \\
\hline $\begin{array}{l}\text { Véhicule personnel } \\
\text { ou bus interurbain }\end{array}$ & $\begin{array}{l}\text { forte } \\
\text { locale et globale } \\
\text { (zone urbaine ou faiblement urbanisée, } \\
\text { franchissement des frontières }\end{array}$ & $\begin{array}{l}\text { petit nombre, } \\
\text { essentiellement LFNs, } \\
\text { plusieurs technologies d'accès }\end{array}$ & $\begin{array}{c}\text { handovers verticaux } \\
\text { multidomiciliation }\end{array}$ \\
\hline Train ou bus urbain & $\begin{array}{l}\text { moyenne } \\
\text { locale (à l'intérieur } \\
\text { d'un réseau d'accès } \\
\text { propriétaire) }\end{array}$ & $\begin{array}{l}\text { quelques dizaines ou centaines } \\
\text { en majorité VMNs } \\
\text { (appartenant aux passagers) } \\
1 \text { ou } 2 \text { technologies d'accès }\end{array}$ & $\begin{array}{c}\text { contrôle d'accès } \\
\text { handovers horizontaux } \\
\text { multidomiciliation } \\
\text { mobilité enchaînée }\end{array}$ \\
\hline Avion & $\begin{array}{l}\text { faible, } \\
\text { locale (réseau appartenant aux } \\
\text { opérateurs de satellite et aéroports) }\end{array}$ & $\begin{array}{l}\text { quelques dizaines ou centaines } \\
\text { en majorité VMNs } \\
2 \text { ou } 3 \text { technologies d'accès } \\
\text { (satellite, radio, wi-fi) }\end{array}$ & $\begin{array}{l}\text { sécurité renforcée } \\
\text { contrôle d'accès } \\
\text { handovers verticaux } \\
\text { mobilité enchaînée } \\
\text { multidomiciliation }\end{array}$ \\
\hline PAN & $\begin{array}{l}\text { faible (selon l'usage) } \\
\text { zone urbaine }\end{array}$ & $\begin{array}{l}\text { petit nombre } \\
\text { plusieurs technologies d'accès }\end{array}$ & $\begin{array}{l}\text { connectivité globale } \\
\text { handovers verticaux }\end{array}$ \\
\hline
\end{tabular}

Tableau 1. Les caractéristiques par type

\begin{tabular}{|l|l|c|}
\hline Besoins des Utilisateurs & Besoins du Système & Type \\
\hline grand nombre de réseaux mobiles & IPv6 & F, D \\
grand nombre d'équipements & & F, D \\
\hline interconnexion des équipements & réseau embarqué & $\mathrm{F}$ \\
\hline accès continu à Internet & $\begin{array}{l}\text { support des réseaux mobiles } \\
\text { technologies d'accès multiples }\end{array}$ & $\mathrm{F}$ \\
& multidomiciliation & F,D \\
& handovers horizontaux /verticaux & F,P \\
\hline équipements standards et & Fransparence de la mobilité pour LFNs & $\mathrm{D}$ \\
faible coût des équipements & (compatibilité avec l'existant) & \\
\hline connecter téléphone mobile, PDA & support stations mobiles & $\mathrm{F}$ \\
connecter PAN & support mobilité enchaînée & $\mathrm{F}$ \\
\hline qualité, performance & handovers rapides & $\mathrm{P}$ \\
& faible coût de la signalisation & $\mathrm{P}$ \\
\hline routage optimal & $\mathrm{P}$ \\
\hline maintenance, facilité d'utilisation & auto-configuration & $\mathrm{F}, \mathrm{D}$ \\
\hline sécurité & contrôle d'accès & $\mathrm{F}$ \\
& (autorisation, authentification) & $\mathrm{F}$ \\
& confidentialitée & $\mathrm{F}$ \\
\hline
\end{tabular}

Tableau 2. Les besoins : fonctionnel $(\mathbf{F})$, de performance $(\mathbf{P})$, de déploiement $(\mathbf{P})$

Le tableau 1 résume le type de mobilité, d'équipement et les besoins correspondant à certains usages des réseaux mobiles. Le tableau 2 résume les fonctions requises selon les besoins de l'utilisateur.

\section{Les travaux de l'IETF}

Que ce soit une station qui se déplace ou un MR avec le réseau qui lui est attaché, le problème est relativement similaire. Cependant, à la problématique habituelle du changement d'adresse s'en ajoute d'autres propres aux réseaux mobiles. Nous commençons donc par analyser l'aptitude de Mobile IPv6 (i.e. la solution pour le support des stations mobiles) à supporter la mobilité des réseaux. Nous présentons ensuite les étapes qui ont conduit à la création d'un nouveau groupe de travail à l'IETF pour traiter le cas spécifique des réseaux mobiles, et nous détaillons la solution recommandée par cette organisation, ainsi que les problèmes qui subsistent. 


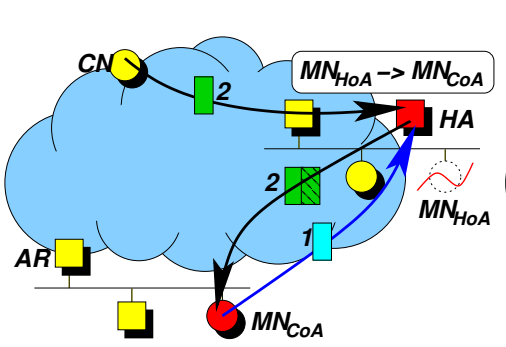

(a) Premiers paquets de données

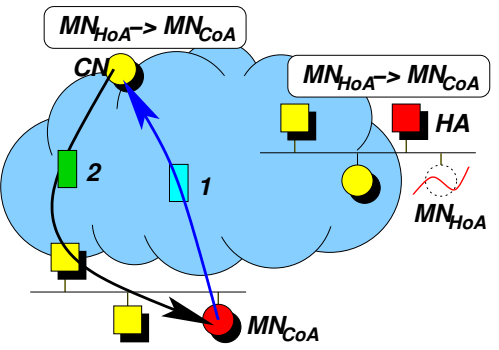

(b) Routage optimal

Figure 2. Mobilité des stations avec Mobile IPv6

\subsection{Mobile IPv6 : support de la mobilité des stations}

Mobile IPv6 (Johnson et al., 2004) gère le problème de la mobilité en allouant deux adresses à chaque station mobile MN. La première (Home Address ou $M N_{H o A}$ ) est une adresse permanente qui identifie la station dans le sous-réseau mère. La seconde (Care-of Address ou $M N_{C o A}$ ) est temporaire et est obtenue dans le sous-réseau visité sur lequel le mobile prend ancrage. Une relation (binding) est établie entre les deux adresses, ce qui permet d'utiliser la première comme identificateur, et la seconde pour le routage. Le MN peut posséder simultanément plusieurs adresses $M N_{C o A}$ sur des liens différents, mais il n'en enregistre qu'une seule, appelée l'adresse temporaire primaire. Le mobile fait ensuite parvenir l'adresse temporaire primaire $M N_{C o A}$ au moyen d'un message de mise-à-jour (BU), non seulement à son agent mère (HA), mais aussi à chacun de ses correspondants CNs. Les BUs sont des paquets spéciaux contenant deux extensions d'en-tête IPv6 supplémentaires. L'adresse permanente est contenue dans l'option Home Address Option de l'entête d'extension Destination Option. Le message de mise-à-jour instruisant le destinataire d'ajouter ou de mettre à jour l'entrée correspondante dans son cache (Binding Cache) est contenu dans l'entête d'extension Mobility Header.

Au début d'une communication entre un correspondant $\mathrm{CN}$ et un mobile $\mathrm{MN}$, le $\mathrm{CN}$ n'a pas connaissance de l'adresse temporaire $M N_{C o A}$. Il envoie donc les paquets normalement vers l'adresse $M N_{H o A}$ du mobile. Ces paquets sont ainsi routés jusqu'au sous-réseau ayant le même préfixe que l'adresse de destination et parviennent donc sur le sous-réseau mère du mobile. Le paquet y est intercepté par le HA puis encapsulé vers $M N_{C o A}$ comme cela est montré sur la figure 2a. A la réception d'un paquet encapsulé, le mobile le décapsule, ajoute l'adresse du CN dans sa liste de correspondants et peut lui envoyer un $\mathrm{BU}$ (figure $2 \mathrm{~b}$ ) pour permettre un routage optimal. Lorsque le $\mathrm{CN}$ obtient un $\mathrm{BU}$ valide (i.e. obéissant aux tests de conformité liés à la sécurité, particulièrement l'authentification de l'émetteur par son destinataire), une nouvelle entrée est ajoutée dans son cache et les paquets suivants peuvent être envoyés di- 
rectement à l'adresse $M N_{C o A}$ (c'est-à-dire sa localisation topologique effective sans passer par le HA) en utilisant une extension d'en-tête IPv6 (Routing extension header) contenant l'adresse $M N_{H o A}$ pour l'identification du mobile. En recevant un paquet contenant cet entête, la couche IP du mobile permute l'adresse destination $M N_{C o A}$ et l'adresse $M N_{H o A}$ contenue dans l'option et passe le paquet à la couche supérieure. Pour en savoir plus sur le fonctionnement de Mobile IPv6 et les protocoles qui lui sont associés, nous recommandons (Soliman, 2004), ouvrage paru récemment.

\subsection{Support de la mobilité des réseaux avec Mobile IPv6}

Mobile IP, dans sa version IPv4 (Perkins, 2002), mentionne brièvement les réseaux mobiles. En effet, les concepteurs de Mobile IPv4 pensent gérer la mobilité des réseaux de manière similaire à celle des stations, mais ceci est présenté de manière très succincte, en partant de l'observation qu'un réseau mobile n'est autre qu'un réseau rattaché à un routeur mobile, c'est-à-dire un nœud mobile comme une autre (voir (Perkins, 2002) section 4.5, (Perkins, 1998) section 5.12, (Solomon, 1998) section 11.2). A chacun de ses déplacements, il suffirait donc au MR d'obtenir une adresse temporaire $M R_{C o A}$ et de l'enregistrer auprès de son HA comme dans le cas d'une station mobile. La solution semble d'autant plus simple avec Mobile IPv4 que tous les paquets de données passent nécessairement par le HA dans les deux sens; il n’y a donc pas d'optimisation de routage ce qui simplifie la solution.

Cette analyse n'a cependant pas été suffisamment poussée par leurs auteurs jusqu'à considérer les caractéristiques et les besoins spécifiques à la mobilité des réseaux détaillés dans la section 4. De plus, la version IPv6 de Mobile IP ne fait plus mention du support des réseaux mobiles. Il s'est en effet avéré que Mobile IPv6 n'est pas adapté au support de la mobilité des réseaux comme cela a été démontré dans (Ernst, 2001). D'une part, la spécification ne permet pas de rediriger les paquets destinés aux nœuds situés derrière le MR, et d'autre part le mécanisme d'optimisation du routage est inadéquat :

- Maintien des sessions : le besoin le plus essentiel est le maintien des sessions ouvertes entre les MNNs et leurs CNs lors des déplacements. Un MR opérant Mobile IPv6 enverrait alors $M R_{C o A}$ à son HA. Or, si le MR se limite à cette fonction de Mobile IPv6, rien n'indique au HA qu'il doit procéder à l'encapsulation vers $M R_{C o A}$ pour l'ensemble des MNNs situés dans le réseau mobile. En fait, seuls les paquets dont la destination finale est le MR (i.e. son adresse $M R_{H o A}$ ) peuvent être encapsulés. Les sessions établies entre MNNs et CNs ne peuvent donc pas être maintenues.

- Optimisation du routage : comme tous les paquets transmis entre MNNs et leurs CNs transitent nécessairement par un MR, le changement de point d'ancrage du seul MR a un impact sur le routage vers l'ensemble des MNNs. Ceux-ci peuvent donc sembler mobiles du point de vue des CNs. En revanche, la structure interne d'un réseau mobile est préservée lors des déplacements du MR. Pour permettre un routage optimal en appliquant les mécanismes de Mobile IPv6, les CNs se devraient de recevoir périodiquement un BU contenant l'adresse $M R_{C o A}$. L'envoi périodique de BUs 


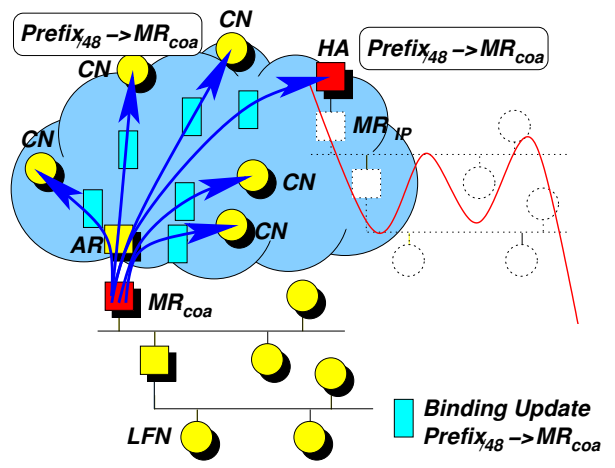

Figure 3. Explosion des messages de contrôle (Binding Updates)

à chaque $\mathrm{CN}$ provoquerait alors une explosion de BUs (Binding Update Explosion ou $B U$ Storm) comme cela est montré sur la figure 3. Ceci se traduit à la fois par un coût très élevé en ressource mémoire pour garder l'historique des BUs envoyés aux différents CNs, en CPU pour l'envoi des BUs et en bande passante en particulier à proximité de $\mathrm{AR}$ où les liens pourraient être congestionnés par un nombre excessif de messages de contrôle.

- Sécurisation de la signalisation : d'autre part, l'envoi des BUs vers les CNs incomberait alors au nœud obtenant l'adresse temporaire, donc au MR. Or, il n'est pas possible d'effectuer le test d'authentification de la provenance des BUs tel qu'il est effectué par les CNs dans Mobile IPv6. Le BU étant émis par le MR, le CN devrait être en mesure de réaliser ce test pour les paquets destinés à l'adresse $M R_{H o A}$, mais rien ne lui permettrait de déduire qu'il doit en faire autant pour tous les nœuds qui partage le même préfixe que $M R_{H o A}$. Cela nécessiterait un nouveau mécanisme. Pour pallier à ces problèmes, les MNNs pourraient envoyer directement les BUs à leurs propres $\mathrm{CNs}$, mais cela nécessiterait également des modifications dans la spécification, entre autres un mécanisme pour que les MNNs détectent la mobilité du réseau et obtiennent l'adresse temporaire du MR. Ceci n'est pas souhaitable car la gestion de la mobilité du réseau ne se ferait plus de manière transparente pour les MNNs. D'autre part, on constaterait une possible duplication des BUs dans le cas où un $\mathrm{CN}$ correspondrait avec plus d'un MNN du même réseau mobile, ce qui consommerait inutilement de la bande passante et de la mémoire dans le cache de ce $\mathrm{CN}$.

De nombreux problèmes subsistent et Mobile IPv6 ne peut pas être simplement étendu pour supporter les réseaux mobiles. Le support des réseaux mobiles nécessite donc une solution spécifique, mais dont le concept n'est pas forcément très éloigné de Mobile IPv6, du moins est-ce l'idée soutenue par l'IETF, bien qu'il existe d'autres approches, décrites dans la section 6 . 


\subsection{Le groupe de travail NEMO de l'IETF}

La problématique des réseaux mobiles a fait sommairement son apparition à l'IETF à plusieurs reprises avant de véritablement prendre son envol à partir de 2000 lorsque l'analyse de Mobile IPv6 décrite dans la section précédente a été soumise au groupe de travail Mobile IP. À partir de ce moment, la communauté IETF a pris conscience du besoin de traiter cette problématique explicitement, tout en évitant d'interférer avec le développement de Mobile IP. Après de longues négociations, et trois réunions de l'IETF, la communauté est parvenue à s'entendre sur une charte, ce qui a donné naissance en octobre 2002 au nouveau groupe de travail $\mathrm{NEMO}^{7}$. Les contours de la problématique ont été difficiles à établir notamment à cause de la confusion souvent faite entre réseaux mobiles, réseaux cellulaires et réseaux ad hoc (voir section 4), et aussi à cause de la méconnaissance de la complexité de l'optimisation du routage et de son impact sur la sécurité.

Le groupe de travail NEMO a décidé d'aborder le problème en deux étapes afin de produire une solution déployable rapidement et de contourner les problèmes de sécurité liés à l'optimisation du routage :

- Support de base (NEMO Basic Support) : dans un premier temps, le groupe se doit de standardiser une solution simple, connue sous le nom NEMO Basic Support, permettant de maintenir les sessions pour l'ensemble des MNNs, sans modifications des MNNs, sans optimisation de routage, et définie sur le modèle Mobile IPv6 (voir la description détaillée section 5.4). Outre la spécification du protocole, les livrables du groupe NEMO comprennent quelques spécifications annexes telles la délégation des préfixes, une MIB, les modèles d'usage du sous-réseau mère (voir le document home network models (Thubert et al., 2006)), et une analyse de la problématique des configurations multidomiciliées (voir section 5.5).

- Support étendu (NEMO Extended Support) : dans un second temps, le groupe se doit d'étudier les problèmes d'optimisation. Les livrables sont des documents de synthèse décrivant la problématique de la multidomiciliation ( $\mathrm{Ng}$ et al., 2006a), celle de l'optimisation du routage ( $\mathrm{Ng}$ et al., 2005) et ses approches potentielles ( $\mathrm{Ng}$ et al., 2006b), ne reposant pas nécessairement sur le modèle Mobile IPv6. A l'issue de ces documents, le groupe devra décider soit de continuer ses travaux dans le but de standardiser une ou plusieurs solutions pour l'optimisation du routage, soit de déclarer sa fermeture. Dans le premier cas, la charte doit préalablement être redéfinie, à la vue des conclusions des documents de synthèse. Ces problèmes complexes justifient en fait l'approche en deux étapes décidée par le groupe NEMO. Nous reviendrons sur ces problèmes de recherche dans la section 5.5.

7. Groupe de travail NEMO de l'IETF : http ://www.ietf.org/html.charters/nemo-charter.html, nommé ainsi non pas en mémoire du capitaine Némo qui pilote le Nautilus mais bien entendu en référence à la transcription anglaise de la mobilité des réseaux : NEtwork MObility. 


\subsection{Description technique du support de base}

Le protocole dit Support de Base (NEMO Basic Support, RFC 3963) (Devarapalli et al., 2005) est une solution permettant le seul maintien des sessions en procédant à la redirection des paquets destinés aux MNNs vers la position courante du MR. Elle représente le consensus des personnes impliquées dans le groupe de travail et rassemble l'ensemble des diverses propositions qui y ont été faites lors de sa création. Elle est établie sur le modèle de Mobile IPv6 selon des règles préalablement édictées par le groupe de travail dans un document dressant la liste des fonctions requises ((Ernst, 2005), section 4). La règle fondamentale est de ne pas imposer de modifications sur les MNNs.

Le principe de base de la solution est que tous les nœuds du réseau mobile partagent le (ou les) même préfixe d'adresse IP (MNP). Le déplacement du MR ne causant pas de changement du point d'ancrage physique des MNNs, seul le (ou les) MR est tenu de changer l'adresse de son interface externe. En revanche, les MNNs conservent leur adresse. Le changement de point d'ancrage est donc géré de manière transparente pour les MNNs afin de ne pas nécessiter de fonctionnalités nouvelles dans l'ensemble des implémentations IPv6. La solution consiste donc à établir un tunnel bidirectionnel entre le HA et le MR.

Comme dans Mobile IPv6, le support de base gère le problème de la mobilité en allouant deux adresses à chaque interface externe du MR (ou des MRs dans le cas où il y en aurait plusieurs). La première (Home Address ou $M R_{H o A}$ ) est une adresse permanente qui identifie le MR dans le sous-réseau mère. Elle identifie soit l'interface externe et a pour préfixe celui du sous-réseau mère, soit l'interface interne du MR (voir (Thubert et al., 2006)), et elle a pour préfixe MNP comme chacun des MNNs du même réseau mobile. La seconde (Care-of Address ou $M R_{C o A}$ ) est temporaire et est obtenue dans le sous-réseau visité sur lequel l'interface externe du MR prend ancrage. Le protocole établit ainsi une relation entre le préfixe MNP utilisé comme identificateur, et l'adresse temporaire $M R_{C o A}$, utilisée pour le routage. Seuls les MRs qui changent leur point d'ancrage obtiennent cette nouvelle adresse, les autres MNNs conservent leur seule adresse $M N N_{M N P}$; la gestion de la mobilité leur est ainsi transparente.

Le MR fait ensuite parvenir l'adresse temporaire primaire $M R_{C o A}$ au moyen d'un message de mise-à-jour des préfixes (PBU) à son agent mère (HA). Les PBUs IPv6 sont des paquets spéciaux contenant une entête d'extension Mobility Header. Lorsque HA reçoit un PBU valide (i.e. obéissant aux tests de conformité liés à la sécurité, particulièrement l'authentification de l'émetteur par son destinataire), l'entrée correspondante au MNP est ajoutée ou mise à jour dans son cache (Binding Cache). Elle instruit le HA d'encapsuler les paquets à destination d'une adresse ayant un préfixe correspondant au MNP (i.e. l'ensemble des stations résidant dans le réseau mobile) vers la destination effective du MR (i.e. $M R_{C o A}$ ).

Lors d'une communication entre un $\mathrm{MNN}$ et un $\mathrm{CN}$, le $\mathrm{CN}$ n'a pas connaissance de l'adresse de routage temporaire $M R_{C o A}$. Les paquets sont donc envoyés normale- 


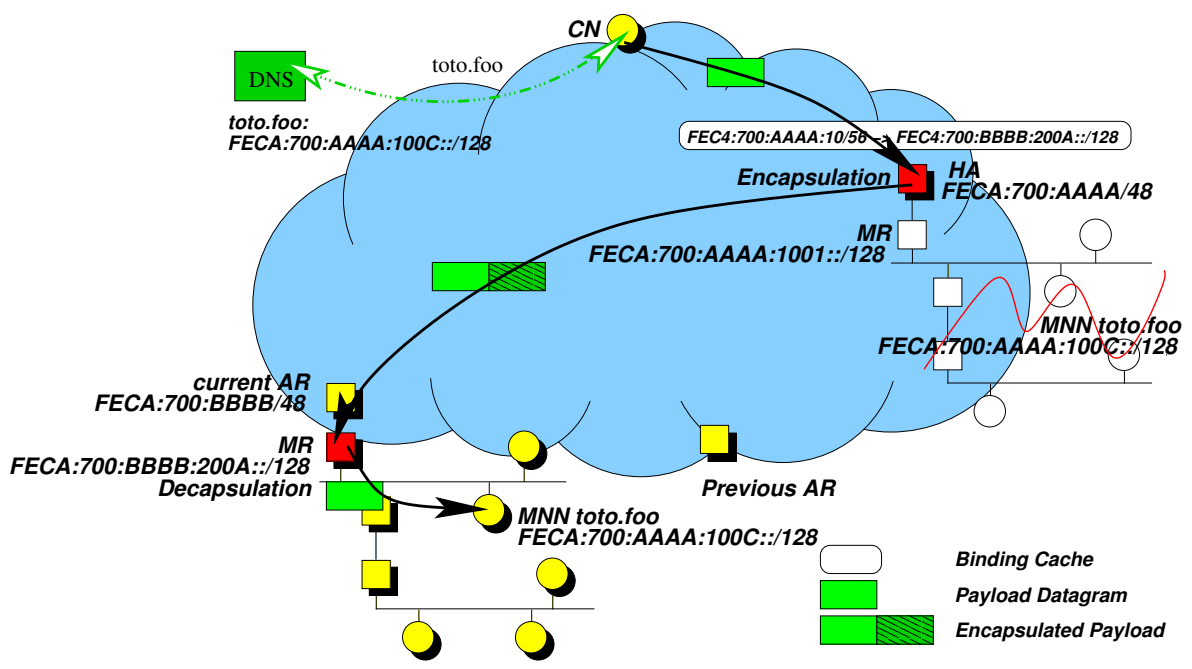

Figure 4. NEMO Support de Base : Tunnel HA-MR

ment vers l'adresse $M N N_{M N P}$ du MNN et routés jusqu'au sous-réseau ayant pour préfixe MNP. Ils parviennent ainsi dans le sous-réseau mère du MR. Les paquets y sont interceptés par le HA puis encapsulés vers $M R_{C o A}$ comme cela est montré sur la figure 4. A la réception d'un paquet encapsulé, le MR le décapsule et le transmet sur son interface interne. Le paquet que reçoit le MNN ne contient donc plus $M R_{C o A}$; l'opération lui est ainsi transparente. Dans le sens inverse, les paquets sont également encapsulés du MR à son HA.

\subsection{Problèmes à résoudre à l'IETF}

Le support de base pose certains problèmes qui sont débattus avec plus ou moins de vigueur sur la liste de discussion du groupe NEMO :

- Multidomiciliation : l'analyse du support de base dans les configurations multidomiciliées ( $\mathrm{Ng}$ et al., 2006a) produite par le groupe de travail NEMO propose dans un premier temps un taxinomie des configurations possibles (voir section 4) avant de présenter la problématique. Plus d'une dizaine de problèmes, plus ou moins complexes, y sont recensés. Le groupe NEMO doit à présent décider lesquels il a vocation à résoudre, les autres (en particulier ceux du filtrage des adresses, de la sélection des adresses, et de la détection des pannes) restant au soin d'autres groupes de travail. Parmi ceux propres à NEMO, nous notons la synchronisation des HAs et MRs, et le support des interfaces multiples. Dans ce dernier cas, le MR peut posséder simultanément plusieurs adresses $M R_{C o A}$ sur des liens différents, mais la spécification ne permet (pour le moment) de n'en enregistrer qu'une seule dans le cache du HA pour 
un MNP donné. La spécification n'interdit cependant pas d'apporter les extensions nécessaires. Les solutions imaginées pour la gestion des interfaces multiples dans Mobile IPv6, en particulier celles de (Montavont, 2004; Wakikawa, 2004) peuvent s'appliquer au cas des réseaux mobiles. Une thèse (Paik, 2004) entièrement consacrée à la mobilité des réseaux contient un chapitre dédié à la sélection du MR. D'autres travaux traitent de problèmes de multidomiciliation liés à la mobilité enchainée : comment découvrir la profondeur du réseau ou comment déterminer le root-MR qui connecte le réseau agrégé à Internet (Montavont et al., 2004; Cho et al., 2004; Thubert et al., 2004).

- Transition IPv4/IPv6 : la transition entre IPv4 et IPv6, et la traversée des NATs et PATs causent certains problèmes de compatibilité ; ceux-ci sont actuellement débattus dans les listes de discussions de l'IETF (Soliman et al., 2006).

- Contrôle d'accès : les mécanismes de contrôle d'accès ont été développés pour autoriser et gérer l'accès d'une station dans un réseau quelconque, mais pas pour permettre l'accès à un MR et au réseau qui lui est attaché. Il est nécessaire de mettre en place une procédure permettant aux VMNs de bénéficier de l'accès qu'a obtenu le MR. Ces aspects ont été étudiés dans (Ng et al., 2002; Zrelli et al., 2005; Bournelle et $a l ., 2006)$ mais les problèmes soulevés n'ont pas été jugés spécifiques au cas des réseaux mobiles et ne sont donc pas traités dans le groupe de travail NEMO. Ils doivent pourtant être résolus pour permettre un déploiement commercial du RFC 3963.

- Routage sous-optimal : le transit des paquets par le HA (dans les deux sens) a pour conséquence d'accroître la distance, donc les délais de transmission et l'usage de la bande passante, en particulier lorsque CNs et MNNs sont relativement proches dans la topologie. Ce problème est bien connu dans le domaine du support de la mobilité des stations. Mais il est plus complexe dans NEMO à cause de la multiplicité des configurations possibles (mobilité enchaînée, hétérogénéité des MNNs). Dans le cas d'une configuration dite enchaînée, le défaut d'optimisation de routage se traduit par plusieurs niveaux d'encapsulation et, non pas un routage triangulaire comme dans le cas des stations mobiles (cf. paragraphe précédent), mais un routage quadrilatéral (deux niveaux de mobilité) voire pire, les paquets transitant par le HA de chaque routeur ou station mobile de la hiérarchie. Par exemple, sur la figure 1, l'envoi des paquets de $\mathrm{CN}$ à VMN se traduirait par deux encapsulations, en passant par $H A_{V M N}$ puis $H A_{M R}$. D'autre part, deux VMNs situés dans le même réseau mobile risquent de voir leurs paquets dirigés vers le HA du MR ou leurs HAs respectifs. Dans un tel contexte, la question de l'optimisation du routage devient cruciale. Plusieurs documents existent là aussi pour décrire ces problèmes, en particulier ceux du groupe NEMO précédemment cités ((Ng et al., 2005) pour la problématique et (Ng et al., 2006b) pour l'analyse des solutions) dans lequels le lecteur avisé trouvera une important source de références.

\section{Gestion de la mobilité des réseaux et optimisation du routage}

Dans la section précédente, nous avons parlé de l'approche suivie par l'IETF, emprise plus par des soucis de coût du déploiement que d'efficacité, et qui vise donc à 
imposer un minimum de changements. Il existe cependant une multitude d'approches, dont certaines mériteraient une étude approfondie.

Il n'est pas possible d'établir une liste exhaustive des solutions proposées pour supporter la mobilité des réseaux et permettant l'optimisation du routage, car cellesci sont déjà nombreuses. Elles peuvent néanmoins être regroupées par catégories. La taxinomie que nous proposons ci-dessous est largement inspirée par l'étude des solutions permettant la mobilité des stations. Il nous paraît en effet naturel de faire un parallèle entre la mobilité des réseaux et celle des stations à cause des similitudes de la problématique et des approches existantes. Une telle étude, dont les résultats sont développés dans (Ernst, 2001) (chapitres 3, 4 et 5) montre que le problème d'adressage et de routage peut être résolu de plusieurs manières, et pas seulement selon le modèle double adressage de Mobile IP :

- Double adressage : le moyen le plus répandu, qui est aussi celui préconisé pour l'instant par l'IETF et donc celui adopté pour les standards (Mobile IP, NEMO), fait usage de deux adresses. L'une est permanente et utilisée en tant qu'identifiant d'interface; l'autre est temporaire et est utilisée en tant qu'identifiant de la position dans la topologie et donc pour le routage. Un mécanisme est alors nécessaire pour maintenir à jour la relation entre les deux identifiants. C'est l'approche la plus évidente et la plus simple car elle ne remet pas en cause la pérennité des différents protocoles de la couche réseau et ne nécessite l'ajout de fonctionnalités qu'aux seules entités mobiles (au sens IP du terme) ainsi que dans un routeur situé dans le sous-réseau mère (e.g. le HA). (Ng et al., 2005) dresse une analyse de celles qui tombent dans une approche basée sur Mobile IPv6.

- Routage : cette approche permet de conserver les adresses IP et de laisser la mise-à-jour de la localisation au soin d'un protocole de routage (e.g. OSPF ou BGP4), mais ceux-ci ne sont généralement pas adaptés à des déplacements nombreux ou fréquents car les tables de routage devraient être recalculées en permanence. Une telle approche peut en revanche s'appliquer au cas de l'aéronautique. Le service opérationnel Connexion de Boeing, basé sur une solution BGP-4 propriétaire, dans IPv4, en offre un parfait exemple. Cette approche est aussi suivie par les protocoles de routage ad hoc, ou par ceux de gestion de la mobilité locale des stations (par exemple Cellular IP) et pourraient s'appliquer aux réseaux mobiles.

- Renumérotation : cette approche discutée à l'IETF consiste à changer les adresses de l'ensemble des nœuds de manière à disposer d'une adresse routable topologiquement correcte. Le routage est donc intrinsèquement optimal. En revanche, il faut un mécanisme complémentaire pour ne pas rompre les sessions ouvertes ainsi qu'un mécanisme permettant aux CNs de déterminer l'adresse courante du MNN (un DNS dynamique).

- Séparation des fonctions de l'adresse : cette approche consiste à séparer les deux fonctions d'identification et de localisation des adresses IP de manière à ne plus rompre les sessions lors des déplacements. Par exemple, LINA (Ishiyama et al., 2001) propose d'utiliser la partie haute et variable des adresses pour la localisation, tandis que la partie basse sert à l'identification. Cette solution initialement proposée pour 
la gestion de la mobilité des stations (LIN6) est maintenant étendue au support de la mobilité des réseaux (Oiwa et al., 2003).

- Approche hiérarchique : afin de limiter la signalisation nécessaire à la gestion de la mobilité et de permettre le passage à l'échelle, de nombreuses solutions proposent également de différencier la mobilité locale (entre sous-réseaux topologiquement proches dans la topologie Internet) de la mobilité globale (entre sous-réseaux topologiquement éloignés dans la topologie Internet). Une approche hiérarchique peut alors être utilisée en combinant deux classes de solutions, par exemple le double adressage pour la mobilité globale et un protocole de routage pour la mobilité locale. HMIPv6 (Soliman et al., 2005) est une solution hiérarchique utilisant une approche de double adressage basée sur Mobile IPv6 à la fois pour la mobilité locale et pour la mobilité globale dont le but est de restreindre l'envoi de BUs à une zone locale. Le mobile obtient en fait deux CoAs, l'une (CoA locale) est mise à jour à chacun de ses déplacements, et l'autre (CoA régionale) n'est mise à jour que lors du changement de domaine. Le concept a été étendu aux réseaux mobiles dans une version intermédiaire de 2001, mais cette partie a été retirée de la spécification courante.

- Diffusion multipoint : cette approche permet de lutter contre le phénomène de l'explosion des messages de mise-à-jour en les envoyant à un groupe multicast au lieu de les envoyer à un nœud particulier. Partant du fait que tous les CNs reçoivent des messages de contrôle au contenu identique, (Ernst, 2001) propose une telle solution combinant des extensions multipoint avec le support de base. Une technique multicast dite traditionnelle et reposant sur les protocoles de routage habituels traite du problème du passage à l'échelle lorsque les CNs sont nombreux. Le but consiste à établir un arbre de distribution entre le routeur mobile et le groupe multipoint constitué par l'ensemble des correspondants du réseau mobile, en vue d'y transmettre les paquets de contrôle permettant le routage optimal. Une seconde approche plutôt destinée à un nombre assez restreint de correspondants, repose sur une nouvelle technique de routage multipoint qui consiste à enregistrer la liste des correspondants directement dans le message de contrôle (XCAST), ce qui évite l'établissement de l'arbre, généralement coûteux, mais limite aussi le nombre de correspondants dû à l'accroissement de la taille du paquet.

- Réseau virtuel : cette approche consiste à positionner quelques routeurs à des emplacements stratégiques, tels les routeurs raccordant chaque domaine à l'Internet, de manière à former un réseau dit overlay de routeurs spécialisés dans la gestion de la mobilité. ORC (Wakikawa et al., 2003; Wakikawa, 2004), par exemple, propose une solution de ce type qui s'apparente en fait à une gestion hiérarchique semblable à HMIPv6. Watari (2005) traite de la question de la mobilité enchaînée et propose quant à lui le déploiement d'un router correspondant dans le site du CN. 


\section{Projets et implémentations de NEMO Basic Support}

Plusieurs implémentations de NEMO Basic Support sont en cours de réalisation ou de déploiement au sein de grands projets publics ou par des industriels. En voici quelques uns :

- ICAR (anciennement InternetCAR) : le scénario décrit dans la section 2 est mis en œuvre sur la plate-forme de démonstration de ce projet basé à Keio University près de Tokyo, et conduit au sein de l'organisation WIDE (Widely Interconnected Distributed Environment). Le projet a été lancé en 1996 dans le but d'étudier le système de communication nécessaire aux applications ITS (entre le véhicule et Internet). Ce projet a suivi l'évolution des travaux de l'IETF depuis ceux du groupe de travail Mobile IP jusqu'à ceux du groupe de travail NEMO. Le projet s'intéresse particulièrement au support de la multidomiciliation. Plusieurs phases du projet ont été réalisées. Dans un premier temps, les études ont porté sur Mobile IPv4, en ne considérant qu'une seule station mobile (un ordinateur muni d'une carte 802.11 et d'un téléphone cellulaire). Les expériences ont gagné en complexité jusqu'à considérer Mobile IPv6 puis NEMO, mais sur un seul véhicule. La première solution présentée à l'IETF pour le support des réseaux mobiles (Prefix-Scope Binding Updates - PSBU) (Ernst, 2001) a ainsi été implémentée et testée sur un véhicule prototype munis de plusieurs technologies d'accès (Ernst et al., 2003). Le réseau mobile déployé dans le véhicule est constitué de trois sous-réseaux isolés les uns des autres. L'un est utilisé pour transmettre les données de contrôle des organes primaires du véhicule (moteur, freins, pression des pneumatiques, et divers capteurs); l'autre pour contrôler les organes moins importants (l'ouverture des portes, des fenêtres), et le troisième pour la transmission des données multimédia. PSBU a ensuite fait place à la solution de l'IETF. Actuellement, le projet développe un réseau test d'accès sans fil sur l'itinéraire d'une ligne de bus. 4 types de capteurs IPv6 ont été développés (température, vitesse, accélération, GPS). Une démonstration a été réalisée lors de l'ITS World Congress de Nagoya au Japon en octobre 2004, où un réseau mobile était déployé dans un bus. ICAR est un des contributeurs essentiels du consortium InternetITS en ce qui concerne le système de communication IPv6. Il bénéficie ainsi des plates-formes de test développées par ce consortium qui impliquent, pour certaines expériences, plusieurs centaines de taxis en activité. Les taxis, à l'exception d'un prototype, n'ont jusqu'à présent pas été équipés d'un MR, en revanche ils ont permis la collecte d'informations permettant d'étudier le trafic et la pertinence des technologies IPv6.

- Nautilus6 ${ }^{8}$ : ce projet franco-japonais a été créé en février 2003, initialement au sein de WIDE (il rassemble à présent Keio University, University of Tokyo, IIJ du côté japonais et l'INRIA, l'ULP, l'ENST-B, l'INT et France Telecom R\&D du côté français), et a pour but de démontrer la faisabilité d'un déploiement de la mobilité dans IPv6. Ceci passe par le déploiement de la mobilité des stations et des réseaux, et

8. Nautilsu6 : http ://www.nautilus6.org. Le lecteur devrait être à présent convaincu qu'un Internet ne peut pas être déployé de manière omniprésente sans que NEMO le soit à l'intérieur des véhicules, bateaux y compris. 
nécessite d'apporter une solution aux besoins soulevés dans la section 4 (connectivité permanente et transparente, performance, sécurité, contrôle d'accès, etc.). Nautilus6 dispose de deux implémentations de NEMO Basic Support. La première (nommée SHISA) tourne sous FreeBSD 4.11 / NetBSD 1.6.2 et a été réalisée en collaboration avec le projet KAME. La deuxième implémentation (NEPL), sous Linux, est basée sur la pile MIPL 2.0 et est réalisée en collaboration avec Go-Core et USAGI ${ }^{9}$. Le projet est très présent à l'IETF et travaille aussi sur les mécanismes de multidomiciliation, d'optimisation des handovers, et d'adaptation du flux de données émis par les applications en fonction du type d'accès disponible. Des démonstrations publiques des mécanismes NEMO et de ses usages potentiels sont réalisées en direct sur Internet (voir le site web) par le biais des plates-formes E-Bike et E-Wheelchair (Ernst, 2004). Des capteurs IPv6 existants ou en cours de réalisation permettront de déterminer les pulsations cardiaques, la localisation par GPS, la température, la vitesse et l'accélération, et un système de vidéo-conférence permettra quant à lui d'envoyer une vidéo de la scène ou de communiquer avec les tiers. Le tout peut être placé sur un vélo ou sur un fauteuil roulant, derrière un routeur mobile opérant NEMO Basic Support. Le MR dispose d'un accès $802.11 \mathrm{~b}$ ou d'un accès cellulaire (AirH, B-Mobile, ou FOMA).

- Overdrive ${ }^{10}$ : ce projet est financé par la Communauté européenne (Programme IST, $5^{\text {th }}$ Framework), dont le but est de développer les mécanismes IPv6 de gestion de la mobilité (réseaux hétérogènes, mobilité des sources, mobilité des routeurs, mécanismes d'autorisation et d'authentification). Il implique notamment Daimler Chrysler, Motorola, France Telecom, Ericsson, et l'Université de Surrey. (Lach et al., 2003; Petrescu et al., 2004) donne quelques détails de l'expérience menée avec NEMO Basic Support, implémenté dans la pile LIVSIX ${ }^{11}$ tournant sous Linux 2.4. Les configurations testées sont celles de la mobilité enchaînée et celle de la multidomiciliation.

- ISO TC204 WG16 : l'ISO conduit d'important travaux de standardisation, notamment dans les technologies de l'information appliquées à l'automobile (TC204). L'architecture CALM a ainsi été définie par le groupe de travail $16^{12}$ qui travaille spécifiquement sur la partie permettant aux véhicules munis de cette architecture CALM de communiquer avec l'infrastructure fixe par le biais d'IPv6. NEMO Basic Support est ainsi considéré pour gérer le changement de point d'ancrage. Les différents CPUs de contrôle devant être isolés des applications multimédia, un modèle tout-IPv6 n'est pour le moment pas envisageable. Ces CPUs de contrôle ne devraient donc pas être munis d'une interface IP. Un sous-réseau de type MOST (bus non-IP) est ainsi préconisé pour ses qualités de transmission temps-réel et sa fiabilité. Par contre les applications non sensibles, en particulier le multimédia, tourneront bien sous IPv6.

9. KAME (à présent défunt) et USAGI sont deux autres projets de WIDE dont l'objectif est l'implémentation d'un pile IPv6 de référence respectivement sous NetBSD/FreeBSD et Linux. Go-Core est un projet de Helsinki University of Technology (HUT).

10. OVERDRIVE : Spectrum Efficient Uni-and Multicast Services Over Multi Radio Networks in Vehicular Environments : http ://www.comnets.rwth-aachen.de/ o_drive/

11. LIVSIX, an open IPv6 source stack by Motorola Labs : http ://www.nal.motlabs.com/livsix 12. ISO/TC204 Transport Information and Control Systems (TICS) WG16 Wide Area Communications/Protocols and Interfaces : http ://www.sae.org/technicalcommittees/tc204wg16.htm 
- Autres projets : Renault a réalisé des démonstrations d'applications ITS sous IPv6 en partenariat avec Cisco en utilisant leur implémentation NEMO Basic Support (Cisco 3200). Il existe bien entendu de nombreux autres projets traitant de NEMO, entre autres DAIDALOS ${ }^{13}$, un autre projet Européen (Programme IST, 6th Framework) et le projet eMOTION ${ }^{14}$ en Australie. L'OTAN, la NASA, Boeing, Panasonic, sont connus pour travailler sur le sujet, certains d'entre eux disposant de leur propre implémentation.

\section{Conclusion}

Dans cet article, nous avons présenté le principe de la mobilité des réseaux, leurs usages, leur problématique et leur prise en charge dans IPv6. Nous avons dressé l'inventaire des contributions dans ce nouveau domaine.

La mobilité des réseaux a ouvert une brèche dans la gestion habituelle de la mobilité et mis à jour de nouveaux problèmes qui nécessiteront de plus amples travaux de recherche. En effet, le besoin de déplacer des réseaux est perçu depuis un certain temps, mais aucun travail significatif ne leur avait été consacré avant les premiers travaux introduits à l'IETF qui ont comblé ce manque. Les problèmes qui leurs sont propres n'ayant pas été abordés, donc suffisamment bien détaillés, les premières discussions à l'IETF ont eu pour résultat une certaine prise de conscience, assez faible dans un premier temps, mais grandissante depuis, dans les rangs de la communauté, qui a abouti avec la création du groupe de travail NEMO. Le support des réseaux mobiles est à présent un sujet qui intéresse de nombreux industriels, allant des fournisseurs d'équipement réseau ou d'électronique grand public jusqu'aux fabriquants d'automobiles, en passant par les opérateurs de téléphone et de transport public.

Le support de la mobilité des réseaux permet de développer l'idée d'un Internet omniprésent, à tout instant, à tout endroit, avec n'importe qui. Les applications multimédia seront les premières à bénéficier de ce type d'environnement. En effet, la mobilité des réseaux rend en pratique possible la mobilité de tout équipement, ce qui implique aussi que toute application doit être en mesure de fonctionner dans un environnement mobile. Ceci nécessitera des mécanismes de changement d'interface et de routeur mobile, et la prise en considération d'un certain nombre de paramètres, en particulier le changement de qualité de service, de bande passante, de règles d'usage (pare-feu) en fonction des technologies accessibles à un instant donné et du réseau d'accès

La gestion de la mobilité des réseaux mobiles doit faire face à de nombreuses contraintes. Tout d'abord, il convient de supporter les réseaux mobiles en nombre et en taille importantes, en considérant divers types de configurations (un seul sous-réseau, la multidomiciliation, la mobilité enchaînée). Le nombre élevé de correspondants nous impose de minimiser la quantité de messages de contrôle relatifs à la gestion de la

13. DAIDALOS : http ://www.ist-daidalos.org

14. eMOTION : http ://www.cse.unsw.edu.au/ ocean/emotion/ 
mobilité tout en optimisant le routage. Ces messages doivent être échangés en toute sécurité et authentifiés par leurs destinataires pour s'assurer qu'ils ne sont pas envoyés par un usurpateur. Les mécanismes intrinsèques de Mobile IP doivent être revus pour permettre un routage optimal tout en considérant la question de la mobilité enchâ̂née et de la multidomiciliation qui accentuent encore plus la question du passage à l'échelle. La question de l'optimisation de routage n'est pour l'instant pas officiellement abordée par le groupe de travail NEMO. En revanche, il existe déjà quelques documents présentant la problématique et de nombreuses propositions.

\section{Remerciements}

Pour leur aide, conseils et m'avoir permis d'ouvrir le débat sur les routeurs mobiles à l'IETF, je tiens à adresser mes remerciements à mes mentors de thèse Claude Castelluccia (INRIA Rhône-Alpes) et Hong-Yon Lach (Motorola Labs Paris). Certains résultats de cette thèse ont été mis en pratique au sein de l'équipe InternetCAR de Keio University, SFC, Japon, que j'ai par la suite rejoint et dont je remercie les membres. Ceci n'aurait pu se faire sans l'appui de Jun Murai, dit l'Internet samurai, vide-président de Keio University et président de WIDE, avocat hors normes d'IPv6. Je remercie également les relecteurs officiels dont les commentaires ont permis d'améliorer sensiblement cet article ainsi que Thomas Noël pour une dernière relecture.

\section{Bibliographie}

Boot J., « Public Safety Applications for Mobile Networks, and Project MESA », 53rd IETF meeting, Motorola, March, 2002. Presentation Material, MONET BOF.

Bournelle J., Valadon G., Binet D., Zrelli S., Laurent-Maknavicius M., « AAA Considerations Within Several NEMO Deployment Scenarios », 1st International Workshop on Network Mobility (WONEMO), Sendai, Japan, January, 2006. http ://www.icoin.org/wonemo.

Cho H., Paik E., Choi Y., « HMRA : Hierarchical Router Advertisement for Nested Mobile networks », 1st IEEE VTS Asia Pacific Wireless Communications Symposium (APWCS), p. 78-80, January, 2004.

Cizault G., "IPv6 : Théorie et Pratique", n ISBN 284177337X, 4th edn, Editions O'Reilly, November, 2005.

Deering S., Hinden R., Internet Protocol Version 6 (IPv6) Specification, Request For Comments $\mathrm{n}^{\circ}$ 2460, IETF, December, 1998.

Devarapalli V., Wakikawa R., Petrescu A., Thubert P., Network Mobility (NEMO) Basic Support Protocol, Request For Comments n ${ }^{\circ}$ 3963, IETF, January, 2005.

Ernst T., Le Support des Réseaux Mobiles dans IPv6, PhD thesis, Université Joseph Fourier, October, 2001. http ://www.inria.fr/rrrt/tu-0714.html.

Ernst T., « E-Wheelchair : A Communication System Based on IPv6 and NEMO », 2nd International Conference on Smart Homes and Health Telematic (ICOST), Keio University, Japan, Singapore, September, 2004. http ://icost2004.i2r.a-star.edu.sg. 
Ernst T., Network Mobility Support Requirements, Internet Draft $\mathrm{n}^{\circ}$ draft-ietf-nemorequirements-05.txt, IETF, November, 2005. Work in progress.

Ernst T., Lach H.-Y., Network Mobility Support Terminology, Internet Draft $n^{\circ}$ draft-ietf-nemoterminology-05.txt, IETF, March, 2006. Work in progress.

Ernst T., Mitsuya K., Uehara K., « Network Mobility from the InternetCAR Perspective », JOIN : Journal on Interconnection Networks, September, 2003.

Ernst T., Uehara K., « Connecting Automobiles to the Internet», 3rd International Workshop on ITS Telecommunications (ITST), Seoul, South Korea, November, 2002.

Ishiyama M., Kunishi M., Uehara K., Esaki H., Teraoka F., « LINA : A New Approach to Mobility Support in Wide Area Networks », IEICE Transactions on Communications, August, 2001.

Johnson D. B., Perkins C., Arkko J., Mobility Support in IPv6, Request For Comments n 3775, IETF, June, 2004.

Kellerer W., Bettstetter C., Schwingenshlög C., Sties P., « (Auto) Mobile Communication in a Heterogeneous and Converged Wordl », IEEE Personal Communications, vol. 8, ${ }^{\circ} 6$, p. 41-47, December, 2001.

Lach H.-Y., Janneteau C., Leinmueller T., Olivereau A., Petrescu A., Leinmueller T., Wolf M. M., Pilz M., Laboratory and Field Experiments with IPv6 Mobile Networks in Vehicul Environments, Internet Draft $\mathrm{n}^{\circ}$ draft-lach-nemo-experiments-overdrive-01.txt, IETF, October, 2003. Work in progress.

Manner J., Kojo M., Mobility Related Terminology, Request For Comments $n^{\circ} 3753$, IETF, June, 2004.

Montavont N., Multiple Interfaces Management and Fast Handover Mechanisms on an IPv6 Terminal (Gestion Optimisée d'Interfaces Multiples et Prise en Compte des Déplacements Rapides sur un Terminal IPv6 Mobile), PhD thesis, Université Louis Pasteur, Strasbourg, France, Département Informatique, LSIIT, UMR CNRS-ULP 7005, September, 2004.

Montavont N., Ernst T., Noel T., « Multihoming in Nested Mobile Networks », International Symposium on Applications and the Internet (SAINT) - IPv6 : Technology and Deployment Workshop", Tokyo, Japan, January, 2004.

Ng C., Paik E., Ernst T., Bagnulo M., Analysis of Multihoming in Network Mobility Support, Internet Draft $n^{\circ}$ draft-ietf-nemo-multihoming-issues-06, IETF, June, 2006a.

Ng C., Tanaka T., Usage Scenario and Requirements for AAA in Network Mobility Support, Internet Draft $\mathrm{n}^{\circ}$ draft-ng-nemo-aaa-use-00.txt, IETF, October, 2002. Expired.

Ng C., Thubert P., Watari M., Zhao F., Network Mobility Routing Optimization Problem State-

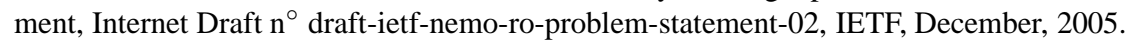

Ng C., Zhao F., Watari M., Thubert P., Network Mobility Routing Optimization Solution Space Analysis, Internet Draft $n^{\circ}$ draft-ietf-nemo-ro-space-analysis-02, IETF, February, 2006b.

Oiwa T., Kunishi M., Ishiyama M., Kohno M., Teraoka F., « A Network Mobility Protocol based on LIN6 », IEEE VTC2003 Fall IP Mobility, October, 2003.

Paik E., Performance Enhancement for IPv6 Network Mobility, PhD thesis, Seoul National University, School of Electrical Engineering and Computer Science, August, 2004.

Partridge C., Technical Criteria for Choosing IP the Next Generation (IPng), Request For Comments n ${ }^{\circ}$ 1726, IETF, December, 1994. 
Perkins C., IP Mobility Support, Request For Comments n³ 3344, IETF, August, 2002. Obsoletes RFC3220 and RFC2002.

Perkins C. E., Mobile IP, Design Principles and Practices, Wireless Communications Series, Addison-Wesley, 1998. ISBN 0-201-63469-4.

Petrescu A., Lach H.-Y., Janneteau C., Wolf M., Leinmuller T., Barz C., Pilz M., Frank M., Tonjes R., «IPv6-based OverDRiVE Moving Networks : Mobility Management and Testbed Implementation », IST Mobile Summit, Lyon, France, June, 2004.

Quinot T., "An IPv6 architecture for Aeronautical Telecommunication Network », Master's thesis, Ecole Nationale Supérieure des Télécommunications Paris, EUROCONTROL - European Organization for the Safety of Air Navigation - ISA project (IPv6, Satellite communication and ATMode for ATN), 1998. http ://www.eurocontrol.fr/.

Robert O., « IPv6 in the Sky », 1st International Global IPv6 Summit, Eurocontrol Experimental Centre, October, 1999. Presentation Material.

Soliman H., Mobile IPv6, Mobility in a Wireless Internet, Addison-Wesley, 2004. ISBN 0-20178897-7.

Soliman H., Castelluccia C., El-Malki K., Bellier L., Hierarchical Mobile IPv6 Mobility Management (HMIPv6), Request For Comments n 4140, IETF, August, 2005. Experimental.

Soliman H., Tsirtsis G., Devarapalli V., Kempf J., Levkowetz H., Thubert P., Wakikawa R., Mobile IPv6 Support For Dual Stack Hosts and Routers (DSMIPv6), Internet Draft ${ }^{\circ}$ draftietf-mip6-nemo-v4traversal-02.txt, IETF, June, 2006. Work in progress.

Solomon J. D., Mobile IP, The Internet Unplugged, Prentice Hall Series in Computer Networking and Distributed Systems, Prentice Hall PTR, 1998. ISBN 0-13-856246-6.

Tanenbaum A. S., Computer Networks - Third Edition, Prentice-Hall International, Inc, 1996.

Terry D., " Mobile Network in Aircraft : Concept of Operation », "Mobile Network Conops" thread in the NEMO mailing list at the IETF, Boeing, August, 2004. http ://www1.ietf.org/mail-archive/web/nemo/current/msg01427.html.

Thubert P., Montavont N., Nested NEMO Tree Discovery, Internet Draft $\mathrm{n}^{\circ}$ draft-thubert-treediscovery-01.txt, IETF, October, 2004. Work in progress.

Thubert P., Wakikawa R., Devarapalli V., NEMO Home Network Models, Internet Draft $\mathrm{n}^{\circ}$ draft-ietf-nemo-home-network-models-06, IETF, February, 2006. Work in progress.

Wakikawa R., Design of Architecture and Protocols for Universal Mobile Internet, PhD thesis, Keio University, Japan, Graduate School of Media and Governance, March, 2004.

Wakikawa R., Koshiba S., Uehara K., Murai J., « ORC : Optimized Route Cache Management Protocol for Network Mobility », IEEE 10th International Conference on Telecommunication (ICT), Tatiti Papeete, French Polynesia, February, 2003.

Watari M., « A Route Optimization Scheme for Nested Mobile Networks », Master's thesis, Keio University, Japan, Graduate School of Media and Governance, March, 2005.

Zrelli S., Ernst T., Bournelle J., Valadon G., Binet D., « Access Control Architecture for Nested Mobile Environments in IPv6», 4th Conference and Security and Network Architecture (SAR), Batz-sur-Mer, France, June, 2005. http ://www-lor.int-evry.fr/sar05.

Thierry Ernst a effectué ses études doctorales à l'INRIA au sein du projet PLANETE. Sa thèse portait sur la mobilité des réseaux et a servi de base à la mise en place du groupe de travail 
NEMO de l'IETF dont il est le fondateur et le co-animateur. Il a ensuite rejoint le laboratoire du Professeur Jun Murai à Keio University au Japon d'où il a mis en place le projet Nautilus6 traitant de la mobilité IPv6. Il est récemment retourné à l'INRIA au sein du projet IMARA qui travaille sur les véhicules intelligents

Article reçu le 22/04/2004 Version révisée le 04/04/2005 
ANNEXE POUR LE SERVICE FABRICATION

A FOURNIR PAR LES AUTEURS AVEC UN EXEMPLAIRE PAPIER

DE LEUR ARTICLE ET LE COPYRIGHT SIGNE PAR COURRIER

LE FICHIER PDF CORRESPONDANT SERA ENVOYE PAR E-MAIL

1. ARTICLE POUR LA REVUE:

RSTI - TSI. Volume $25-n^{\circ}$ 5/2006

2. AUTEURS :

Thierry Ernst

3. TITRE DE L'ARTICLE :

Le support des réseaux mobiles dans IPv6

4. TITRE ABRÉGÉ POUR LE HAUT DE PAGE MOINS DE 40 SIGNES :

Les réseaux mobiles dans IPv6

5. DATE DE CETTE VERSION :

25 juillet 2006

6. Coordonnées des auteurs :

- adresse postale :

INRIA Rocquencourt, Domaine de Voluceau B.P. 105

78153 Le Chesnay Cedex FRANCE

thierry.ernst@inria.fr

- téléphone : +33139635930

- télécopie : +33139635491

- e-mail : thierry.ernst@inria.fr

7. LOGICIEL UTILISÉ POUR LA PRÉPARATION DE CET ARTICLE :

LATEX, avec le fichier de style article-hermes2.cls,

version $1.23 \mathrm{du} 17 / 11 / 2005$.

8. FORMULAIRE DE COPYRIGHT :

Retourner le formulaire de copyright signé par les auteurs, téléchargé sur :

http://www.revuesonline.com

SERVICE ÉDITORIAL - HERMES-LAVOISIER

14 rue de Provigny, F-94236 Cachan cedex

Tél. : 01-47-40-67-67

E-mail : revues@lavoisier.fr

Serveur web : http://www.revuesonline.com 